\title{
Regulation of hepatic mitochondrial oxidation by glucose-alanine cycling during starvation in humans
}

\author{
Kitt Falk Petersen, ${ }^{1,2}$ Sylvie Dufour, ${ }^{1,2}$ Gary W. Cline, ${ }^{1,2}$ and Gerald I. Shulman ${ }^{1,2,3}$ \\ 'Department of Medicine, ${ }^{2}$ Yale Diabetes Research Center, and ${ }^{3}$ Department of Cellular \& Molecular Physiology, Yale School of Medicine, New Haven, Connecticut, USA.
}

\begin{abstract}
In order to determine whether the glucose-alanine cycle regulates rates of hepatic mitochondrial oxidation in humans, we applied positional isotopomer NMR tracer analysis (PINTA) to assess rates of hepatic mitochondrial oxidation and pyruvate carboxylase flux in healthy volunteers following both an overnight (12 hours) and a 60-hour fast. Following the 60-hour fast, rates of endogenous glucose production and mitochondrial oxidation decreased, whereas rates of hepatic pyruvate carboxylase flux remained unchanged. These reductions were associated with reduced rates of alanine turnover, assessed by $\left[{ }^{-13} \mathrm{C}\right]$ alanine, in a subgroup of participants under similar fasting conditions. In order to determine whether this reduction in alanine turnover was responsible for the reduced rates of hepatic mitochondrial oxidation, we infused unlabeled alanine into another subgroup of 60-hour fasted subjects to increase rates of alanine turnover, similar to what was measured after a 12hour fast, and found that this perturbation increased rates of hepatic mitochondrial oxidation. Taken together, these studies demonstrate that $\mathbf{6 0}$ hours of starvation induce marked reductions in rates of hepatic mitochondrial oxidation, which in turn can be attributed to reduced rates of glucose-alanine cycling, and reveal a heretofore undescribed role for glucose-alanine in the regulation of hepatic mitochondrial oxidation in humans.
\end{abstract}

\section{Introduction}

The glucose-alanine cycle represents a critical link between carbohydrate and amino acid metabolism and comprises a series of reactions in which pyruvate, derived mostly from intramyocellular glycolysis, is transaminated with ammonia, derived from muscle protein catabolism, to form L-alanine $(1,2)$. Alanine is subsequently transported to the liver, where the amino group is transferred to $\alpha$-ketoglutarate by alanine transaminase to form glutamate and pyruvate. The amino group, which was transferred from alanine to glutamate, is subsequently converted to urea, and the newly generated pyruvate is converted to glucose. Besides transferring potentially toxic ammonia, derived from muscle protein catabolism, to the liver for conversion to urea, this cycle also transfers energy from the liver to the muscle by the generation of 2 moles of ATP for every mole of glucose that is glycolyzed and recycles 3-carbon units back to the liver for conversion to glucose. However, it is unknown whether the glucose-alanine cycle participates in other critical functions of hepatic metabolism during prolonged (60 hours) starvation.

We have recently demonstrated a critical role for decreased glucose-alanine cycling, due to hepatic glycogen depletion, in promoting marked reductions in rates of hepatic mitochondrial oxidative metabolism in awake rats during prolonged (48 hours) starvation (3).

Related Commentary: p. 4563

Conflict of interest: The authors have declared that no conflict of interest exists. Copyright: @ 2019, American Society for Clinical Investigation.

Submitted: May 14, 2019; Accepted: July 18, 2019; Published: September 23, 2019.

Reference information: / Clin Invest. 2019;129(11):4671-4675.

https://doi.org/10.1172/JCl129913.
Furthermore, we found that reduced rates of hepatic mitochondrial oxidation during prolonged starvation could be attributed to a $40 \%$ reduction in rates of glucose-alanine cycling. However, whether alterations in the rates of glucose-alanine cycles have similar effects in the regulation of hepatic mitochondrial oxidation in humans during starvation is not known.

In order to determine whether starvation promotes reduced rates of hepatic mitochondrial oxidation (rate of citrate synthase flux $\left[\mathrm{V}_{\mathrm{CS}}\right]$ ) in humans, we applied positional isotopomer NMR tracer analysis (PINTA) to assess rates of hepatic $\mathrm{V}_{\mathrm{CS}}$ and pyruvate carboxylase flux $\left(\mathrm{V}_{\mathrm{PC}}\right)$ as well as the hepatic mitochondrial redox state by measuring the ratio of plasma concentrations of $\beta$-hydroxybutyrate to acetoacetate $([\beta-\mathrm{OHB}] /[\mathrm{AcAc}])(4-7)$ in healthy volunteers following a 12-hour overnight fast and again following a 60-hour fast (8). In order to determine whether alanine turnover is reduced during starvation, we assessed rates of $\left[3-{ }^{-13} \mathrm{C}\right]$ alanine turnover in another subgroup of individuals under the same 12-hour and 60-hour fasted conditions. Finally, in order to determine whether decreased rates of alanine turnover during starvation play a causal role in promoting decreased rates of hepatic mitochondrial oxidation, we infused alanine in a subgroup of 60-hour fasted subjects to approximately match rates of alanine turnover in 12-hour fasted subjects and reassessed rates of hepatic $V_{P C}$ and $V_{C S}$ flux by PINTA under these conditions.

\section{Results and Discussion}

Sixty hours of starvation promoted an approximately $25 \%$ reduction in plasma glucose and insulin concentrations, compared with the overnight (12 hours) fast (Table 1 ), which could be attributed to similar reductions in the rates of endogenous glucose production (Figure $1 \mathrm{~A})$. As expected, these changes were associated with marked 
Table 1. Anthropometric data, plasma metabolite, and hormone concentrations in healthy volunteers after 12-hour and 60-hour fasts

\begin{tabular}{|c|c|c|c|}
\hline & $\begin{array}{c}12-\text { Hour fast } \\
(n=15)\end{array}$ & $\begin{array}{c}\text { 60-Hour fast } \\
(n=15)\end{array}$ & $P$ value \\
\hline Age (years) & $26 \pm 2$ & - & \\
\hline Body weight (kg) & $73.8 \pm 3.1$ & $72.1 \pm 3.3$ & 0.0003 \\
\hline $\mathrm{BMI}\left(\mathrm{kg} / \mathrm{m}^{2}\right)$ & $23.6 \pm 0.9$ & $23.1 \pm 0.9$ & 0.0002 \\
\hline Glucose (mmol/L) & $5.01 \pm 0.11$ & $3.73 \pm 0.09$ & $<0.0001$ \\
\hline Lactate (mmol/L) & $0.90 \pm 0.08$ & $1.03 \pm 0.05$ & 0.241 \\
\hline NEFAs (mmol/L) & $0.50 \pm 0.066$ & $1.36 \pm 0.10$ & $<0.0001$ \\
\hline Alanine (mmol/L) & $0.27 \pm 0.02$ & $0.23 \pm 0.02$ & 0.125 \\
\hline Clycine (mmol/L) & $0.19 \pm 0.02$ & $0.15 \pm 0.02$ & 0.051 \\
\hline Serine $(\mathrm{mmol} / \mathrm{L})$ & $0.021 \pm 0.002$ & $0.020 \pm 0.002$ & 0.879 \\
\hline Leucine (mmol/L) & $0.15 \pm 0.01$ & $0.24 \pm 0.01$ & 0.009 \\
\hline Isoleucine (mmol/L) & $0.06 \pm 0.01$ & $0.11 \pm 0.01$ & 0.0001 \\
\hline Aspartate+asparagine (mmol/L) & $0.041 \pm 0.004$ & $0.037 \pm 0.003$ & 0.131 \\
\hline Phenylalanine (mmol/L) & $0.056 \pm 0.004$ & $0.055 \pm 0.003$ & 0.787 \\
\hline Clutamate+glutamine (mmol/L) & $0.51 \pm 0.04$ & $0.46 \pm 0.03$ & 0.145 \\
\hline$\beta$-hydroxybutyrate (mmol/L) & $0.26 \pm 0.11$ & $3.31 \pm 0.30$ & $<0.0001$ \\
\hline $\mathrm{AcAc}(\mathrm{mmol} / \mathrm{L})$ & $0.17 \pm 0.02$ & $0.72 \pm 0.10$ & $<0.0001$ \\
\hline Insulin (pmol/L) & $84 \pm 16$ & $48 \pm 8$ & 0.0173 \\
\hline C-peptide (ng/mL) & $5.32 \pm 0.87$ & $2.80 \pm 0.46$ & 0.009 \\
\hline Glucagon (ng/mL) & $93 \pm 6$ & $132 \pm 11$ & 0.0027 \\
\hline Leptin (ng/mL) & $7.36 \pm 1.57$ & $3.53 \pm 0.40$ & 0.0167 \\
\hline $\mathrm{TSH}(\mathrm{mlU} / \mathrm{mL})$ & $2.66 \pm 0.36$ & $1.01 \pm 0.13$ & 0.0003 \\
\hline Total $\mathrm{T}_{3}$ (ng/dL) & $0.78 \pm 0.07$ & $0.82 \pm 0.05$ & 0.594 \\
\hline Total $\mathrm{T}_{4}(\mu \mathrm{g} / \mathrm{dL})$ & $5.97 \pm 0.26$ & $6.99 \pm 0.36$ & 0.0035 \\
\hline Energy expenditure (Kcal/24 hours) & $1689 \pm 76$ & $1678 \pm 52$ & 0.860 \\
\hline
\end{tabular}

mlU, milli international units.

increases in plasma glucagon and nonesterified fatty acid (NEFA) concentrations, which resulted in an increase in rates of $\beta-\mathrm{OHB}$ production, leading to increased plasma ketone ( $\beta$-OHB and $\mathrm{AcAc}$ ) concentrations (Table 1 ) and an approximately 3 -fold increase in the hepatic mitochondrial redox state, as reflected by an increase in the plasma $[\beta-\mathrm{OHB}] /[\mathrm{AcAc}]$ ratio (Figure $1 \mathrm{~B})$. This reduction in rates of endogenous glucose production could entirely be attributed to reductions in rates of net hepatic glycogenolysis, since rates of hepatic pyruvate carboxylase flux $\left(\mathrm{V}_{\mathrm{PC}}\right)$ remained relatively unchanged during this 12-hour to 60-hour fasted transition (Figure 1C). These findings are consistent with direct measurements of net hepatic glycogenolysis in humans, by ${ }^{13} \mathrm{C}$ magnetic resonance spectroscopy (MRS), under similar fasting conditions, demonstrating that rates of hepatic gluconeogenesis remain relatively constant during this period of starvation and that reductions in rates of net hepatic glycogenolysis can account for virtually all of the reduction in rates of endogenous glucose production during this fasting interval (9). Using PINTA, we also found that 60 hours of fasting promoted an approximately $90 \%$ increase in the $\mathrm{V}_{\mathrm{PC}} / \mathrm{V}_{\mathrm{CS}}$ ratio (Figure $1 \mathrm{D}$ ), which could entirely be attributed to an approximately $50 \%$ reduction in rates of hepatic mitochondrial oxidation $\left(\mathrm{V}_{\mathrm{CS}}\right)$ compared with the 12-hour overnight fasting conditions (Figure 1E). This starvation-induced reduction in rates of hepatic mitochondrial oxidation $\left(\mathrm{V}_{\mathrm{CS}}\right)$ was associated with an approximately $50 \%$ reduction in plas- ma leptin and thyroid-stimulating hormone (TSH) concentrations, but were independent of changes in plasma $\mathrm{T}_{3}$ concentrations or changes in whole body energy expenditure (Table 1 ).

In order to determine whether 60 hours of starvation altered rates of glucose-alanine cycling, we next measured endogenous rates of alanine turnover in a similar group of young, lean individuals following 12 hours and 60 hours of fasting assessed by $\left[3-{ }^{13} \mathrm{C}\right]$ alanine turnover. Rates of alanine turnover in plasma following a 12-hour fast were approximately $425 \mu \mathrm{mol} / \mathrm{min}$ (Figure $1 \mathrm{~F}$ ), which is in good agreement with rates of alanine turnover previously measured in overnight-fasted humans using similar methodology (10). Following 60 hours of fasting, rates of alanine turnover decreased by approximately $30 \%$ (Figure $1 \mathrm{~F}$ ) despite no significant alterations in plasma alanine concentrations (Table 1). This starvation-induced reduction in rates of whole body alanine turnover is consistent with the observed $75 \%$ reduction in net alanine release across the forearm that occurs in humans during prolonged starvation (1). Previous studies in awake rats found that reduced rates of glucose-alanine cycling following 48 hours of starvation were associated with reduced rates of net hepatic glycogenolysis, resulting in decreased rates of hepatic glucose production and reductions of plasma glucose concentrations from approximately $6 \mathrm{mM}$ to approximately $5 \mathrm{mM}$. The major role of hepatic glycogenolysis and concomitant reductions in plasma glucose concentrations in regulating glucose-alanine cycling during starvation was then demonstrated by using a glycogen phosphorylase inhibitor, which inhibited net hepatic glycogenolysis in glycogen-replete 8-hour fasted rats and decreased rates of glucose-alanine cycling to rates observed in hepatic glycogen-depleted 48-hour fasted rats despite unchanged rates in hepatic $V_{P C}$ (3). Consistent with these rodent studies, we found that 60 hours of fasting resulted in marked reductions in rates of glucose-alanine cycling in humans independently of any changes in hepatic $\mathrm{V}_{\mathrm{PC}}$ flux, suggesting that these reductions in glucose-alanine cycling reflect reductions in hepatic glycogen content and corresponding reductions in rates of net hepatic glycogenolysis, as previously observed (9).

In order to determine whether this $30 \%$ reduction in alanine turnover might be playing a causal role in promoting the marked reduction in rates of hepatic mitochondrial oxidation observed following a 60-hour fast, we infused L-alanine in a subgroup of 60-hour fasted individuals to increase rates of alanine turnover to approximately match rates observed in 12-hour fasted subjects and then repeated the PINTA measurements of hepatic pyruvate carboxylase flux, hepatic $\mathrm{V}_{\mathrm{PC}} / \mathrm{V}_{\mathrm{CS}}$, and rates of hepatic mitochondrial oxidation. The L-alanine infusion promoted an approximately $30 \%$ increase in rates of endogenous glucose production $\left(\mathrm{V}_{\mathrm{EGP}}\right)$ (Figure 2A), which could be attributed to an approximately $30 \%$ increase in rates of hepatic pyruvate carboxylase flux $\left(\mathrm{V}_{\mathrm{PC}}\right)$ (Figure 2B). These results demonstrate that hepatic pyruvate carboxylase flux is substrate limited after 60 hours of starvation, despite being activated by increased hepatic acetyl-CoA content, which would be predicted by the 12 -fold increase in rates of $\beta$-OHB turnover (11) and increased plasma glucagon concentrations (Table 1) (12). This increase in hepatic $\mathrm{V}_{\mathrm{PC}}$ flux was associated with an approximately $20 \%$ reduction in the $\mathrm{V}_{\mathrm{PC}} / \mathrm{V}_{\mathrm{CS}}$ ratio (Figure $2 \mathrm{C}$ ) and could entirely be attributed to an approximately $70 \%$ increase in rates of hepatic mitochondrial oxidation $\left(\mathrm{V}_{\mathrm{CS}}\right)$ (Figure 2D). 
A

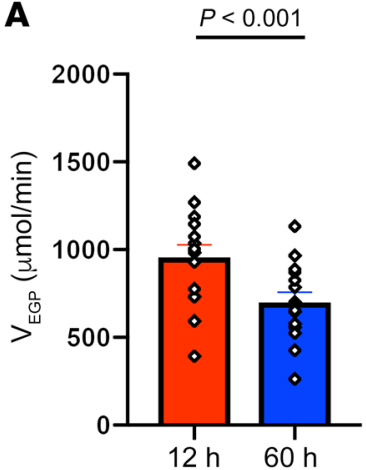

D

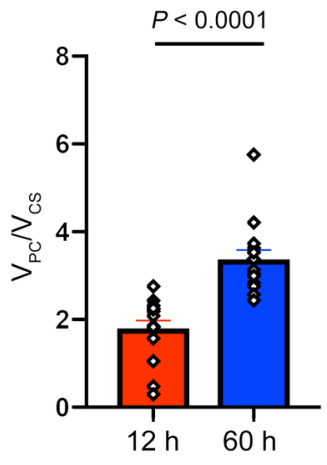

B

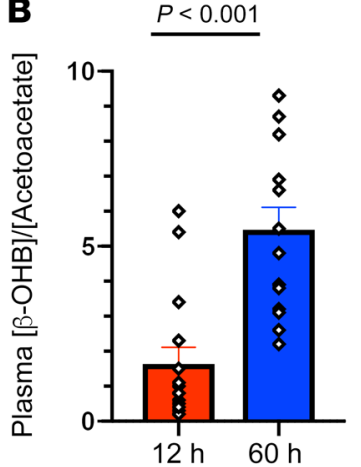

E

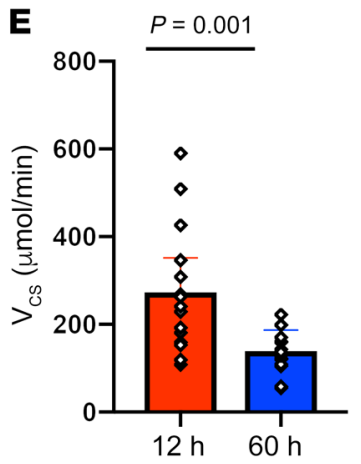

C
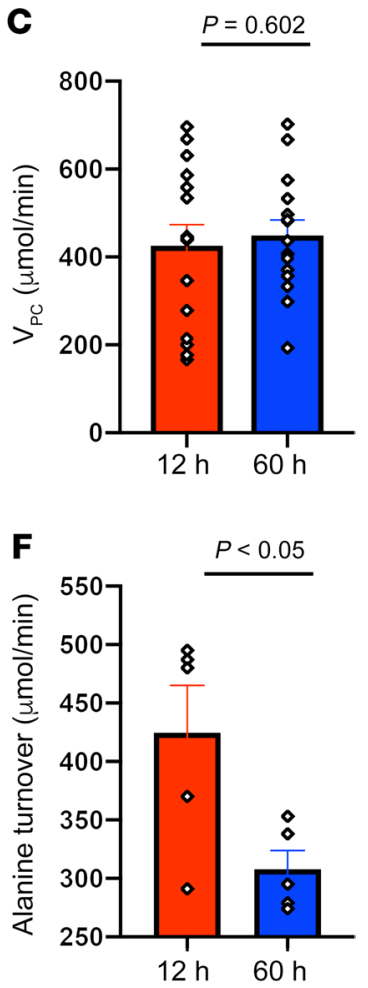

Figure 1. Hepatic glucose and mitochondrial metabolism in healthy, young lean subjects after $\mathbf{1 2}$ hours and $\mathbf{6 0}$ hours of starvation.

(A) Rates of endogenous glucose production $\left(\mathrm{V}_{\mathrm{ECP}}\right)(n=15)$. (B) Ratio of plasma concentrations of $\beta-\mathrm{OHB}$ to plasma concentrations of AcAc $(n=15)$. (C) Rates of hepatic pyruvate carboxylase flux $\left(\mathrm{V}_{\mathrm{PC}}\right)(n=15)$. (D) Relative rates of hepatic pyruvate carboxylase flux vs. citrate synthase flux $\left(\mathrm{V}_{\mathrm{PC}} / \mathrm{V}_{\mathrm{cs}}\right)(n=15)$. (E) Rates of hepatic mitochondrial oxidation $\left(V_{c s}\right)(n=15)$. (F) Rates of alanine turnover in a subgroup of healthy volunteers $(n=5)$. All rates are shown at 12 hours and 60 hours of starvation. Comparisons between the 12-hour and 60-hour fast were performed using a paired, 2-tailed Student's $t$ test, and $P<0.05$ was considered statistically significant. The corresponding plasma enrichments for glucose, lactate, and B-OHB are shown in Supplemental Figures 1, 2, and 3.
We next wanted to understand the potential mechanism by which alterations in glucose-alanine cycling might regulate hepatic mitochondrial oxidation in humans during 60 hours of starvation. Alterations in plasma $\mathrm{T}_{3}, \mathrm{~T}_{4}$, and glucagon concentrations could potentially increase rates of hepatic mitochondrial oxidation, but the increase in hepatic $\mathrm{V}_{\mathrm{CS}}$ with alanine infusion occurred independently of any changes in these hormones (Table 2). It is also possible that alanine-induced increases in hepatic $\mathrm{V}_{\mathrm{PC}}$ flux could have, at least in part, contributed to the observed increase in rates of hepatic mitochondrial oxidation $\left(\mathrm{V}_{\mathrm{CS}}\right)$ during the alanine infusion, by promoting hepatic mitochondrial anaplerosis. However, this does not appear to be a major contributing factor to this process, given that hepatic $\mathrm{V}_{\mathrm{PC}}$ did not decrease substantially during the 60-hour fast, despite marked reductions in rates of hepatic mitochondrial oxidation. This finding is in contrast to our study in rodents (3), in which 48-hour starvation led to marked reductions in both hepatic $\mathrm{V}_{\mathrm{PC}}$ flux and glucose-alanine cycling. These species differences can most likely be attributed to the much higher rates of metabolism in rats, in which 48 hours of fasting leads to virtually total depletion of whole-body fat stores, severe hypoleptinemia $(<1 \mathrm{ng} / \mathrm{mL})$, and hypercorticosteronemia in contrast to the still relatively ample fat stores in 60-hour fasted lean humans. We also assessed hepatic redox potential, as reflected by the ratio of the plasma concentrations of $\beta-\mathrm{OHB}$ to $\mathrm{AcAc}$, and found that the hepatic redox index increased approximately 3.4-fold during 60 hours of starvation and decreased significantly during the alanine infusion (Figure 2E). Increased hepatic redox states have been shown to decrease rates of hepatic mitochondrial oxidation in vitro $(4,5,13)$, and it is possible that an increase in the hepatic redox potential during 60 hours of starvation contributed to the reduced rates of hepatic mitochondrial oxidation and that the alanine infusion promoted an increase in rates of hepatic $\mathrm{V}_{\mathrm{CS}}$ by reducing the hepatic redox state.
Taken together, these studies demonstrate that decreased glucose-alanine cycling is rate controlling in the regulation of hepatic pyruvate carboxylase flux after 60 hours of fasting in humans. Furthermore, they demonstrate that reductions in glucose-alanine cycling during starvation promote reduced rates of hepatic mitochondrial oxidation and reveal a heretofore undescribed role for the glucose-alanine cycle in the regulation of hepatic mitochondrial oxidation in humans. Future studies will be necessary to determine whether alterations in the hepatic mitochondrial redox state and/or other factors are responsible for mediating this skeletal muscle-liver crosstalk. In addition, given recent studies demonstrating a critical threshold for hypoleptinemia to stimulate the hypothalamic-pituitary-adrenocortical axis in both rodents (3) and humans during more prolonged (10 days) starvation (14), which has been shown to modulate white adipocyte lipolysis, hepatic gluconeogenesis (3), and hunger (15) in rodents, it will be of interest to determine the impact of hypoleptinemia and hypercortisolemia on these same parameters in humans.

\section{Methods}

Subjects and study procedures. Twenty healthy, lean, nonsmoking men without history of liver disease, with normal liver function tests, and consuming no more than one alcoholic drink per day were recruited and studied after prescreening with an oral glucose tolerance test (OGTT) to ensure normal glucose tolerance. The PINTA studies were performed in 15 subjects after 12-hour and 60-hour fasts and in 9 of these subjects, who repeated the 60-hour fast with alanine supplementation, as described below.

During the 60-hour fasting period, subjects were instructed to drink plenty of regular water and were contacted daily to ensure compliance, well-being, and to address any issues. Compliance was verified by the fasting blood glucose and ketone concentrations upon admission. All subjects enrolled completed the studies. 

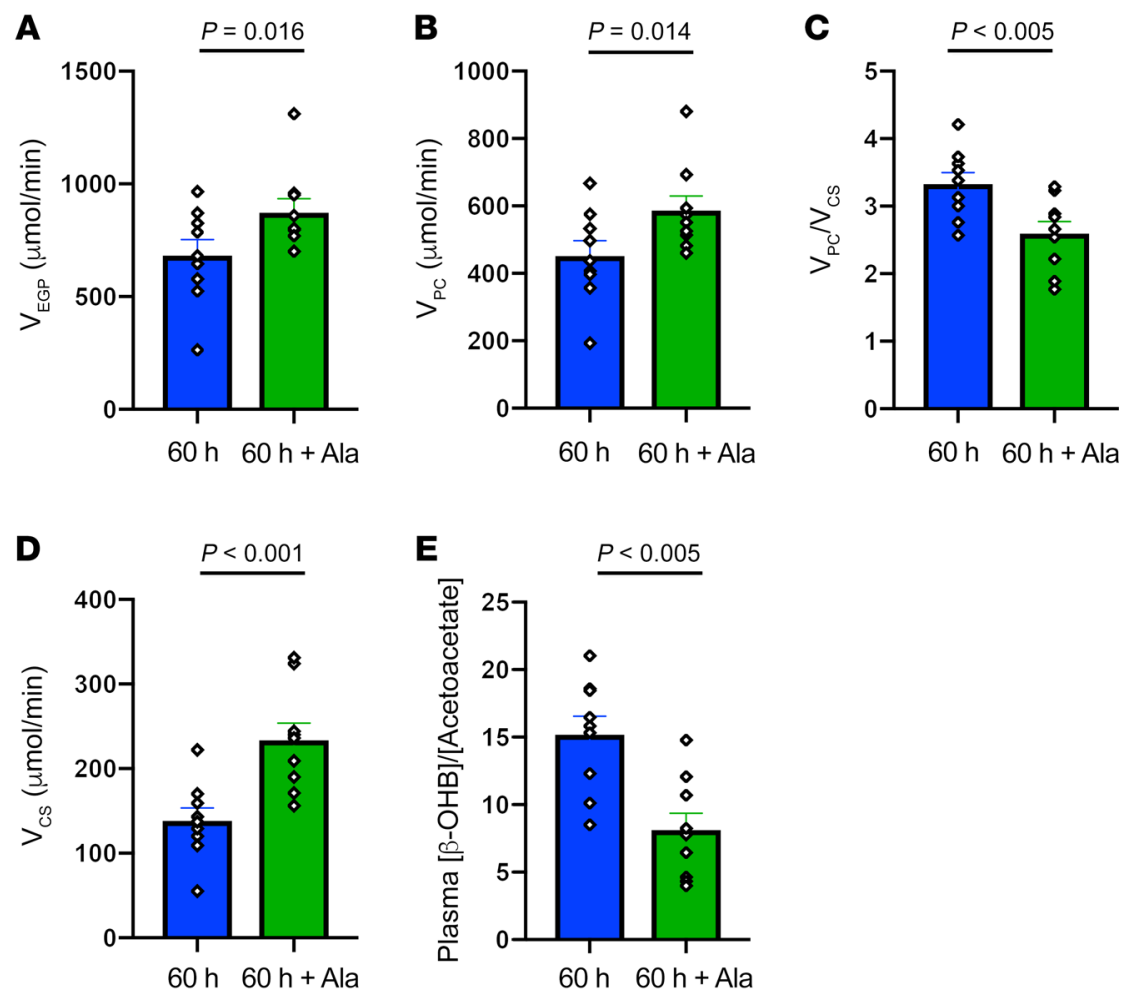

Figure 2. Hepatic glucose and mitochondrial metabolism in healthy, young lean subjects after $\mathbf{6 0}$ hours of starvation and $\mathbf{6 0}$ hours of starvation plus alanine supplementation. (A) Rates of endogenous glucose production $\left(\mathrm{V}_{\mathrm{EGP}}\right)(n=9)$. (B) Rates of hepatic pyruvate carboxylase flux $\left(V_{p c}\right)(n=9)$. (C) Relative rates of hepatic pyruvate and citrate synthase flux $\left(V_{\mathrm{PC}} / \mathrm{V}_{\mathrm{CS}}\right)(n=9)$. (D) Rates of hepatic mitochondrial oxidation $\left(V_{C S}\right)(n=9)$. (E) Relative plasma concentrations of $\beta$-OHB and plasma AcAc $(n=9)$. Comparisons between the 60 -hour fast and 60 -hour fast plus alanine supplementation were performed using a paired, 2-tailed Student's $t$ test, and $P<0.05$ was considered statistically significant. The corresponding plasma enrichments for glucose, lactate, and B-OHB are shown in Supplemental Figures 1, 2, and 3.
After admission at 7:30 am to the Yale Hospital Research Unit (HRU), one intravenous line was inserted into an antecubital vein for infusions and another was inserted retrogradely into a hand vein for blood collections. The hand was warmed in a "hot-box" to "arterialize" the blood, baseline blood samples were collected, and a 3-hour triple-tracer PINTA infusion was started. This consisted of $\left[{ }^{2} \mathrm{H}_{7}\right]$-D-glucose ( $\mathrm{D}_{7}$-glucose, 134 $\mathrm{mmol} / \mathrm{L}$ ), given as a 5 -minute prime of $1.1 \mathrm{mmol} / \mathrm{m}^{2}$ followed by a constant rate of $11.2 \mu \mathrm{mol} /\left(\mathrm{m}^{2}-\mathrm{min}\right),\left[3{ }^{13} \mathrm{C}\right]-\mathrm{Na}-\mathrm{L}-$ lactate $(900 \mathrm{mmol} / \mathrm{L})$, and ${ }^{13} \mathrm{C}_{4}$-Na- $\beta$-OHB $\left({ }^{13} \mathrm{C}_{4}-\beta\right.$-OHB, $\left.15.4 \mathrm{mmol} / \mathrm{L}\right)$, given at constant rates of $4.3 \mu \mathrm{mol} /(\mathrm{kg}-\mathrm{min})$ and $3.2 \mu \mathrm{mol} /\left(\mathrm{m}^{2}-\mathrm{min}\right)$, respectively.

In the 60-hour fast-alanine supplementation PINTA studies, an infusion of unlabeled L-alanine (alanine) was added to the triple-tracer infusion at a constant rate of $5 \mu \mathrm{mol} /(\mathrm{kg}$-min) to increase rates of alanine turnover so that they were similar to what was observed after a 12-hour fast.

In order to determine the amount of L-alanine required for supplementation during the 60 -hour fast, rates of $\left[3-{ }^{-13} \mathrm{C}\right]-\mathrm{Na}-\mathrm{L}$-alanine turnover were measured in a separate group $(n=5)$ of subjects after a 12-hour and a 60-hour fast with a 3-hour infusion of [3-13 $\mathrm{C}]-\mathrm{Na}-\mathrm{L}$-alanine $\left(\left[3{ }^{-13} \mathrm{C}\right]\right.$-alanine $)$ at a constant rate of $0.29 \mu \mathrm{mol} /(\mathrm{kg}-\mathrm{min})$.

In all studies, arterialized blood samples were collected every 10 minutes during the last 40 minutes for gas chromatography mass spectrometry (GC-MS) and hormone and metabolite analyses. In the PINTA studies, a $30 \mathrm{~mL}$ sample was collected at 180 minutes for ${ }^{13} \mathrm{C}$ MRS analyses of positional glucose enrichments using ${ }^{13} \mathrm{C}$ MRS (Bruker Avance III HD, 500 UltraShield NMR Spectrometer, TopSpin 3.2, Bruker) in combination with GC-MS and liquid chromatography-tandem mass spectrometry (LC-MS/MS) analyses as previously described (8). Energy expenditure was measured using indirect calorimetry during the final 30 minutes of each study (Deltratrack Metabolic Monitor, Sensormedics).
Plasma glucose and lactate concentrations were measured using a Yellow Springs STAT 2700 Analyzer (Yellow Springs Instruments). Plasma $\beta$-OHB concentrations were measured by COBAS (Roche Diagnostics). Plasma NEFA was measured using a Wako reagent (Wako Diagnostics). Plasma alanine concentrations were measured by GC-MS: samples were spiked with $\mathrm{a}^{2} \mathrm{H}$-alanine internal standard and prepared for GC-MS using the protocols described above, with the ratio of labeled to unlabeled substrate compared with a standard curve to measure absolute concentrations. Plasma amino acid concentrations (glycine, alanine, serine, leucine, isoleucine, aspartate+asparagine, phenylalanine, and glutamate+glutamine) and $\beta$-OHB enrichments were measured by GC-MS after spiking with ${ }^{2} \mathrm{H}$ or ${ }^{13} \mathrm{C}$ internal standards and derivatization using the protocol described for alanine and as previously described for $\beta$-OHB (11). Plasma immunoreactive insulin, C-peptide, glucagon, leptin, TSH, $\mathrm{T}_{3}$, and $\mathrm{T}_{4}$ concentrations were measured with double-antibody radioimmunoassay techniques using commercially available kits (Linco).

Table 2. Plasma metabolite and hormone concentrations in a subgroup of healthy 60-hour fasted subjects before and after a 3-hour alanine infusion

\begin{tabular}{lccc} 
& $\begin{array}{c}\text { 60-Hour fast } \\
(\boldsymbol{n}=\mathbf{9})\end{array}$ & $\begin{array}{c}\text { 60-Hour fast+alanine } \\
(\boldsymbol{n}=\mathbf{9})\end{array}$ & $\boldsymbol{P}$ value \\
Alanine $(\mathrm{mmol} / \mathrm{L})$ & $0.23 \pm 0.04$ & $0.60 \pm 0.03$ & $<0.0001$ \\
$\beta$-hydroxybutyrate $(\mathrm{mmol} / \mathrm{L})$ & $3.1 \pm 0.3$ & $2.0 \pm 0.4$ & 0.0009 \\
AcAc $(\mathrm{mmol} / \mathrm{L})$ & $0.20 \pm 0.03$ & $0.27 \pm 0.04$ & 0.182 \\
$\mathrm{TSH}(\mathrm{ml} / \mathrm{mL})$ & $0.70 \pm 0.12$ & $0.77 \pm 0.18$ & 0.592 \\
Total $_{3}(\mathrm{mg} / \mathrm{mL})$ & $0.80 \pm 0.04$ & $0.83 \pm 0.07$ & 0.051 \\
Total $_{4}(\mu \mathrm{g} / \mathrm{mL})$ & $6.71 \pm 0.28$ & $5.56 \pm 0.40$ & 0.092 \\
& & & \\
\hline
\end{tabular}


Calculations. Rates of $\mathrm{D}_{7}$-glucose, $\left[{ }^{13} \mathrm{C}_{4}\right] \beta$-OHB, and $\left[3-{ }^{-13} \mathrm{C}\right]$ alanine turnover were calculated during isotopic steady state as the tracer infusion rate $\times$ [(infusate enrichment/plasma enrichment) - 1]. $\mathrm{V}_{\mathrm{PC}} /$ $\mathrm{V}_{\mathrm{EGP}}$ and $\mathrm{V}_{\mathrm{PC}} / \mathrm{V}_{\mathrm{CS}}$ were calculated from the ${ }^{13} \mathrm{C}$ glucose enrichments $\mathrm{m}$ $+1, \mathrm{~m}+2$, and $\left[4-{ }^{13} \mathrm{C}\right]$ glucose, $\left[5^{-13} \mathrm{C}\right]$ glucose, as previously derived (8).

The following equations were used: $\mathrm{V}_{\mathrm{PC}} / \mathrm{V}_{\mathrm{CS}}=\left(\left[5-{ }^{13} \mathrm{C}\right]\right.$ glucose $/(2$ $\times\left[4-{ }^{13} \mathrm{C}\right]$ glucose $\left.)\right)-1$ and $\mathrm{V}_{\mathrm{PC}} / \mathrm{V}_{\mathrm{EGP}}=\mathrm{G} 2 / \mathrm{XFE}^{2}$, where XFE, the fractional triose enrichment, in terms of the ratio of the enrichments of doubly labeled ${ }^{13} \mathrm{C}$-glucose $\mathrm{G} 2$ and singly labeled ${ }^{13} \mathrm{C}$-glucose $\mathrm{G} 1$ was calculated as $\mathrm{XFE}=1 /((1+(1 /(2 \times \mathrm{G} 2 / \mathrm{G} 1)))$.

The key assumptions of mass isotopomer distribution analysis (MIDA) are as follows: (a) a binomial probability analysis describes the distribution of ${ }^{13} \mathrm{C}$-glucose isotopomers resulting from the synthesis of $2{ }^{13} \mathrm{C}$-labeled trioses; (b) there is one gluconeogenic precursor pool enrichment (if not, $\mathrm{V}_{\mathrm{PC}} / \mathrm{V}_{\mathrm{EGP}}$ is underestimated); (c) triose phosphates are equilibrated (if not, $\mathrm{V}_{\mathrm{PC}} / \mathrm{V}_{\mathrm{EGP}}$ is overestimated).

In addition to these assumptions, the MIDA calculation assumes that G2 (i.e., any glucose enriched with $2{ }^{13} \mathrm{C}$ atoms) is due to the condensation of 2 trioses, each labeled with a single ${ }^{13} \mathrm{C}$-atom. However, the use of ${ }^{13} \mathrm{C}$-lactate as the tracer and its subsequent passage through the tricarboxylic acid (TCA) cycle (with entry through PC and pyruvate dehydrogenase $[\mathrm{PDH}]$ ) will lead to trioses with $2{ }^{-13} \mathrm{C}$ atoms. We corrected for any G2 glucose synthesized from ${ }^{13} \mathrm{C}_{2}$-trioses by analysis of the enrichment in the glucose $\mathrm{C} 4-\mathrm{C} 5-\mathrm{C} 6$ fragment. In addition to determining the total $\mathrm{m}+1$ and $\mathrm{m}+2$ enrichment in glucose (GC-MS: CI of glucose pentaacetate, $\mathrm{m} / \mathrm{z} 331 \rightarrow 338$ ), enrichment in the glucose C4-C5-C6 fragment was determined by GC-MS analysis in the EI mode of the glucose aldonitrile pentapropionate derivative by monitoring $\mathrm{m} / \mathrm{z} 259 \rightarrow 265$ (16).

The following equations were also used: $\mathrm{V}_{\mathrm{PC}}=\mathrm{V}_{\mathrm{PC}} / \mathrm{V}_{\mathrm{EGP}} \times \mathrm{V}_{\mathrm{EGP}}$ and $\mathrm{V}_{\mathrm{CS}}$ $=\mathrm{V}_{\mathrm{CS}} / \mathrm{V}_{\mathrm{PC}} \times \mathrm{V}_{\mathrm{PC}}$. The $\mathrm{V}_{\mathrm{PC}} / \mathrm{V}_{\mathrm{CS}}$ ratio was corrected for potential ${ }^{13} \mathrm{CO}_{2}$ labeling as described below. The fractional enrichment of glucose from ${ }^{13} \mathrm{CO}_{2}$ can be increased from PC synthesis of $\left[4-{ }^{13} \mathrm{C}\right]$ oxaloacetate $\left(\left[4-{ }^{13} \mathrm{C}\right] \mathrm{OAA}\right)$ from ${ }^{13} \mathrm{CO}_{2}$ and pyruvate. The labeling of glucose from ${ }^{13} \mathrm{C}$-bicarbonate is dependent upon the relative flux of pyruvate to OAA with equilibration with fumarate and formation of phosphoenolpyruvate (PEP) versus flux of pyruvate to OAA to citrate (i.e., $\mathrm{V}_{\mathrm{PC}} / \mathrm{V}_{\mathrm{CS}}$ ). Only $\left[1{ }^{-13} \mathrm{C}\right] \mathrm{OAA}$ (from the equilibration of $\left[4{ }^{13} \mathrm{C}\right] \mathrm{OAA}$ with fumarate) converted directly to $\mathrm{PEP}$ will label glucose (C3 and $\mathrm{C} 4$ ), since all ${ }^{13} \mathrm{CO}_{2}$ of $\left[4{ }^{-13} \mathrm{C}\right] \mathrm{OAA}$ is lost with flux through the TCA cycle. Hence, the correction of ${ }^{13} \mathrm{CO}_{2}$ follows from sev- eral points: (a) ${ }^{13} \mathrm{CO}_{2}$ will label $\mathrm{C} 4$ of OAA to give $\left[4-{ }^{13} \mathrm{C}\right] \mathrm{OAA}$; (b) $\left[4-{ }^{13} \mathrm{C}\right]$ OAA randomizes to $\left[1-{ }^{13} \mathrm{C}\right] \mathrm{OAA}$ and $\left[4-{ }^{13} \mathrm{C}\right] \mathrm{OAA}$; enrichment in each position is $1 / 2$ of the original $\left[{ }^{13} \mathrm{CO}_{2}\right]$ enrichment; (c) $\left[1-{ }^{13} \mathrm{C}\right] \mathrm{OAA} \rightarrow\left[1{ }^{-13} \mathrm{C}\right] \mathrm{PEP}:$

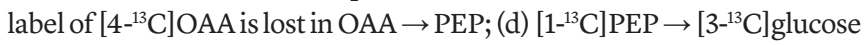
and $\left[4-{ }^{-13} \mathrm{C}\right]$ glucose; (e) ${ }^{13} \mathrm{C}$ of $\left[4-{ }^{13} \mathrm{C}\right] \mathrm{OAA}$ and $\left[1{ }^{-{ }^{13}} \mathrm{C}\right] \mathrm{OAA}$ is lost as ${ }^{13} \mathrm{CO}_{2}$ with $\mathrm{V}_{\mathrm{CS}}$ flux to ${ }^{13} \mathrm{C}$-citrate and first turn of the TCA cycle; ( $\mathrm{f}$ ) therefore, the correction for $\left[{ }^{13} \mathrm{CO}_{2}\right]$ is $2 \times\left[4{ }^{-13} \mathrm{C}\right]$ glucose $-\left({ }^{13} \mathrm{CO}_{2} \times 1 / 2\right) /(2 \times \mathrm{CF})$, where $\left.{ }^{[13} \mathrm{CO}_{2}\right]$ is the liver ${ }^{13} \mathrm{C}$-bicarbonate enrichment, and $\mathrm{CF}=\left(\mathrm{V}_{\mathrm{PC}}+\mathrm{V}_{\mathrm{CS}}\right) / \mathrm{V}_{\mathrm{PC}}$; and $(\mathrm{g}) \mathrm{V}_{\mathrm{PC}} / \mathrm{V}_{\mathrm{CS}}$ corrected for $\left[{ }^{13} \mathrm{CO}_{2}\right]$ was determined iteratively.

Statistics. Statistical analyses were performed with StatPlus software. Statistical comparisons between parameters measured during the 12-hour and 60-hour fast and between the 60-hour fast and 60-hour fast plus alanine supplementation were performed using a paired 2-tailed Student's $t$ test, and a $P$ value of less than 0.05 was considered statistically significant. Percentage changes are calculated from the mean values of the groups. All data are expressed as mean \pm SEM.

Study approval. The Yale Institutional Review Board (Yale University Human Investigation Committee) approved all protocols, and written, informed consent was obtained from each participant after explanation of the purpose, nature, and potential complications of the study. The study was registered at ClinicalTrials.gov (NCT02193295).

\section{Author contributions}

KFP and GIS designed the experiments and wrote the manuscript with assistance from SD and GWC. KFP, SD, and GWC performed the studies. KFP, SD, GWC, and GIS obtained and interpreted data.

\section{Acknowledgments}

The authors would like to thank Anne Impellizeri, Irina Smolgovsky, Mikhail Smolgovsky, Gina Solomon, Xian-Man Zhang, Gina Butrico, Catherine Parmelee, and the Yale HRU for technical assistance and Douglas Rothman for helpful discussions. This work was supported by grants from the NIH (R01 DK113984, R01 NS087568, P30 DK45735, and UL1 RR024139), Gilead Sciences, and the Novo Nordisk Foundation Center for Basic Metabolic Research.

Address correspondence to: Gerald I. Shulman, Yale School of Medicine, TAC S-269, P.O. Box 208020, New Haven, Connecticut 065208020, USA. Phone: 203.785.5447; Email: gerald.shulman@yale.edu.
1. Felig P, Pozefsky T, Marliss E, Cahill GF Jr. Alanine: key role in gluconeogenesis. Science. 1970;167(3920):1003-1004.

2. Felig P. The glucose-alanine cycle. Metabolism. 1973;22(2):179-207.

3. Perry RJ, et al. Leptin mediates a glucose-fatty acid cycle to maintain glucose homeostasis in starvation. Cell. 2018;172(1-2):234-248.e17.

4. Krebs HA, Gascoyne T. The redox state of the nicotinamide-adenine dinucleotides in rat liver homogenates. Biochem J. 1968;108(4):513-520.

5. Williamson DH, Lund P, Krebs HA. The redox state of free nicotinamide-adenine dinucleotide in the cytoplasm and mitochondria of rat liver. Biochem J. 1967;103(2):514-527.

6. Madiraju AK, et al. Metformin suppresses gluconeogenesis by inhibiting mitochondrial glycerophosphate dehydrogenase. Nature. 2014;510(7506):542-546.
7. Madiraju AK, et al. Metformin inhibits gluconeogenesis via a redox-dependent mechanism in vivo. Nat Med. 2018;24(9):1384-1394.

8. Perry RJ, et al. Non-invasive assessment of hepatic mitochondrial metabolism by positional isotopomer NMR tracer analysis (PINTA). Nat Commun. 2017;8(1):798.

9. Rothman DL, Magnusson I, Katz LD, Shulman RG, Shulman GI. Quantitation of hepatic glycogenolysis and gluconeogenesis in fasting humans with 13C NMR. Science. 1991;254(5031):573-576.

10. Perriello G, et al. Estimation of glucose-alaninelactate-glutamine cycles in postabsorptive humans: role of skeletal muscle. Am J Physiol. 1995;269(3 Pt 1):E443-E450.

11. Perry RJ, Peng L, Cline GW, Petersen KF, Shulman GI. A non-invasive method to assess hepatic acetyl-CoA in vivo. Cell Metab. 2017;25(3):749-756.

12. Agius L, Alberti KG. Regulation of flux through pyruvate dehydrogenase and pyruvate carboxylase in rat hepatocytes. Effects of fatty acids and glucagon. Eur J Biochem. 1985;152(3):699-707.

13. Aldakkak M, Stowe DF, Chen Q, Lesnefsky EJ, Camara AK. Inhibited mitochondrial respiration by amobarbital during cardiac ischaemia improves redox state and reduces matrix $\mathrm{Ca} 2+$ overload and ROS release. Cardiovasc Res. 2008;77(2):406-415.

14. Steinhauser ML, et al. The circulating metabolome of human starvation. JCI Insight. 2018;3(16):121434.

15. Perry RJ, et al. Leptin's hunger-suppressing effects are mediated by the hypothalamic-pituitary-adrenocortical axis in rodents. Proc Natl Acad Sci USA. 2019;116(27):13670-13679.

16. Antoniewicz MR, Kelleher JK, Stephanopoulos G. Measuring deuterium enrichment of glucose hydrogen atoms by gas chromatography/mass spectrometry. Anal Chem. 2011;83(8):3211-3216. 